\title{
TV/Series
}

$6 \mid 2014$

Écho et reprise dans les séries télévisées (III) : de la métafiction à la transmédialité

\section{Les amorces narratives, ou le plaisir des histoires cousues de fil blanc}

\section{Marie Tréfousse}

\section{(2) OpenEdition \\ Journals}

Édition électronique

URL : http://journals.openedition.org/tvseries/312

DOI : $10.4000 /$ tvseries. 312

ISSN : 2266-0909

Éditeur

GRIC - Groupe de recherche Identités et Cultures

Référence électronique

Marie Tréfousse, "Les amorces narratives, ou le plaisir des histoires cousues de fil blanc », TV/Series

[En ligne], 6 | 2014, mis en ligne le 01 décembre 2014, consulté le 19 avril 2019. URL : http://

journals.openedition.org/tvseries/312; DOI : 10.4000/tvseries.312

\section{(c) (i) (9)}

TV/Series est mis à disposition selon les termes de la licence Creative Commons Attribution - Pas d'Utilisation Commerciale - Pas de Modification 4.0 International. 


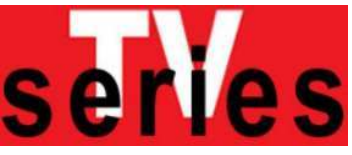

Les amorces narratives, ou le plaisir des histoires cousues de fil blanc

Afin de créer chez le spectateur la nécessité de connaître la suite de la fiction, les feuilletons mettent en place une manipulation très précise, presque mécanique, fondée sur la redondance si souvent reprochée au genre. Dans Éloge de Monte-Cristo, Umberto Eco écrit : "on découvre que les horribles intempérances stylistiques [les redondances] sont, certes, des "chevilles ", mais qu'elles ont une valeur structurale, comme les barres de graphite dans les réacteurs nucléaires, ralentissant le rythme pour rendre nos attentes plus lancinantes, nos prévisions plus hasardeuses ». Par l'étude d'un cas particulier de redondance, l'amorce, nous montrons ici comment les auteurs de feuilletons télévisés manipulent nos attentes et nos prévisions. L'amorce fait partie intégrante des procédés d'anticipation ; sa fonction est de préparer et d'engager la suite. Cette "préparation ", constituée d'une seule ou de plusieurs amorces, a la forme d'un écho, mais un écho "à l'envers ", l'information la plus forte étant la dernière.

Nous nous proposons d'étudier les amorces narratives qui visent à orienter les désirs du spectateur, amorces de courte portée (trouvant leur écho final dans l'épisode), puis amorces portant sur plusieurs épisodes en examinant deux grands classiques : la création du désir que deux personnages entretiennent une relation amoureuse et la création du désir que tel personnage soit l'assassin, ou plutôt «le méchant ». Nous aborderons ainsi la question du besoin de confirmation du spectateur, qui semblerait presque plus fondamental que le besoin d'être surpris. Chaque type d'amorce sera illustré d'un exemple issu de Melrose Place et doublé d'un deuxième exemple issu d'un autre feuilleton afin de vérifier la validité du procédé.

$\mathrm{L}$

'amorce narrative est définie par Gérard Genette dans Figures III. Il la classe aux côtés de l'annonce, dans la catégorie prolepse, c'est-à-dire la catégorie de l'«anachronie par anticipation ${ }^{1}$ » :

L'amorce, à la différence de l'annonce, n'est qu'un « germe insignifiant », et même imperceptible, dont la valeur de germe ne sera reconnue que plus tard, et de façon rétrospective. Encore faut-il tenir compte de l'éventuelle (ou plutôt variable) compétence narrative du lecteur, née de l'habitude, qui permet de déchiffrer de plus en plus vite le code narratif en général, ou propre à tel genre, ou à telle œuvre, et d'identifier les « germes » dès leur apparition ${ }^{2}$.

La terminologie anglo-saxonne, quant à elle, offre deux termes, foreshadowing et telegraphing, que Hallie Ephton définit ainsi : When you insert a hint of what's to come, look at it critically and decide whether it's something the reader will glide right by but remember later with an Aha! That's foreshadowing. If instead the reader groans and guesses what's coming, you've

${ }^{1}$ Gérard Genette, Figures III, Paris, Seuil, 1972, p. 105.

${ }^{2}$ Ibid., p. 113. 
telegraphed. Ultimately, the line between foreshadowing and telegraphing is in the eye of the beholder ${ }^{3}$.

Une amorce, même de type télégraphié, n'est qu'un "germe », un élément implicite qui n'apporte pas de certitude - Hallie Ephton dit que le lecteur " devine », non qu'il est certain. Le caractère implicite de l'amorce est ainsi ce qui la différencie de l'annonce ${ }^{4}$. Par l'amorce, le spectateur est amené à deviner la suite de l'histoire. L'expression populaire à teneur péjorative " cette histoire est cousue de fil blanc » peut donc s'appliquer aux histoires qui contiennent de nombreuses amorces, chaque amorce étant une partie apparente du fil en question.

En étudiant la stratégie mise en place par les amorces, cet article interrogera plus particulièrement les histoires/séries cousues de fil blanc - même si notre corpus n'est pas exempt de feuilletons diffusés sur de prestigieuses chaînes câblées comme HBO et Showtime. Nos exemples seront issus de divers feuilletons mais Melrose Place (FOX, 1992-99) y occupe une place de choix puisqu'il nous servira à montrer qu'un seul et même feuilleton peut réunir différents types d'amorces. Enfin, bien que les amorces soient également présentes dans les séries à épisodes bouclés, notre étude se limitera aux seuls feuilletons. Il sera donc tout d'abord question d'amorces simples (amorce, attente, résolution) qui tendent à mettre un personnage en danger, avec des exemples issus de Plus belle la vie (France 3, 2004-), Game of Thrones (HBO, 2011-), Melrose Place et The L Word (Showtime, 2004-10). Puis, de ces exemples, nous dégagerons les fonctions de l'amorce. Nous nous intéresserons ensuite à ce que nous appellerons des amorces "filées », c'est-à-dire des amorces en série (amorce 1, attente, amorce 2, attente, amorce 3, etc., jusqu'à la résolution) qui peuvent s'étendre sur un grand nombre d'épisodes - ce qui explique aussi notre intérêt exclusif pour le feuilleton. Par le biais des amorces filées, nous étudierons alors deux stratégies récurrentes dans les feuilletons : celle qui consiste à susciter l'envie du spectateur de voir deux personnages vivre une histoire d'amour, avec des exemples issus de Melrose Place et de Life on Mars (ABC, 2008-09), puis celle qui déclenche l'envie qu'un personnage soit "le méchant » (au sens du terme anglo-saxon « villain »), avec pour exemple Melrose Place, Dexter (Showtime 2006-13) et Homeland (Showtime 2011-).

3 Hallie Ephron, Writing and Selling Your Mystery Novel, Writer's Digest Books, 2005, p. 165 .

4 L'annonce est explicite et apporte une certitude. Selon Gérard Genette, «la formule canonique en est généralement un 'nous verrons', ou 'on verra plus tard' », in Figures III, p. 111. 


\section{L'amorce simple : faire peser un danger sur un personnage par une amorce construite sur un hybris}

L'hybris est une notion grecque qui repose sur la démesure et sur le destin. C'est une sorte de péché d'orgueil puni par la destruction (némésis). Traditionnellement, il n'est pas bon pour un personnage de présumer de son avenir ni de la part de bonheur qui lui a été attribué : c'est offenser et outrager les dieux, ce qui appelle un châtiment. Dans les films d'horreur, lorsqu'un personnage dit «I'll be right back » alors que la situation est plus ou moins dangereuse, il signe son arrêt de mort ${ }^{5}$. Dans le feuilleton, l'hybris est également utilisé de deux manières : soit par un outrage direct, c'est-à-dire par une vantardise du type «I'll be right back», soit de façon dégradée sous la forme d'une promesse, d'une interdiction ou d'un conseil. Un exemple d'outrage direct peut se trouver dans l'épisode $\mathrm{n}^{\circ} 1513$ de Plus belle la vie (France 3, 2004-). Vadim apprend une très bonne nouvelle. Il boit une coupe de champagne avec sa femme Luna pour célébrer son succès. Puis, alors qu'elle est sur le point de monter en voiture (le couple doit se rendre à une soirée), Luna lui demande : «Tu es sûr qu'on prend la voiture ? On a bu quand même !? » Vadim répond : " Aujourd'hui, c'est mon jour de chance ; il ne peut rien m'arriver! » Et, quelques minutes plus tard, au moment du cliffhanger, leur voiture se fracasse contre le versant d'une montagne. Dans cet exemple, Vadim " offense les dieux " de façon très explicite. Les amorces impliquent ainsi un fonctionnement en trois temps : mise en germe, attente, résolution.

Le même procédé est à l'oeuvre dans le pilote de Game of Thrones, même si c'est de façon un peu moins sensible : l'outrage n'est pas direct mais dégradé, se présentant sous la forme d'une interdiction mêlée à une promesse. À la minute 15 , la mère, Catelyn, dit à son petit garçon, Bran, qui escalade une muraille : "I want you to promise me, no more climbing ${ }^{6} »$. Bran promet, mal assuré, et, bien que Catelyn lui dise qu'il ment mal, au moment du cliffhanger, Bran tombe d'une muraille ${ }^{7}$. L'hybris étant puni par la destruction, les amorces construites sur l'hybris trouvent systématiquement une résolution à l'inverse : ce ne sera pas le jour de chance de Vadim, et Bran escaladera de nouveau une muraille. Les phrases « aujourd'hui, c'est mon jour de chance, il ne peut rien m'arriver » et « no more climbing » ont l'effet de phrases magiques, de paroles performatives qui fonctionnent $a$

5 Ceci est porté à l'état de théorie dans Scream (Wes Craven, 1996), à la minute 69, lorsque Randy énonce les règles qui permettent à un personnage de survivre dans un film d'horreur.

${ }^{6}$ Catelyn : «Je veux que tu me promettes d'arrêter l'escalade. » (notre traduction, comme pour les autres citations en anglais de cet article)

7 Notons que l'hybris n'est pas du fait de Bran mais de sa mère; la némésis sera donc proportionnelle à sa faute : par sa chute, Bran ne perdra pas la vie mais l'usage de ses jambes. 
contrario et à retardement. Ce retardement - c'est-à-dire le temps d'attente entre l'amorce et sa résolution, que Gérard Genette appelle la portée $^{8}$ de l'amorce - est de longueur variable. Dans les deux exemples cités, la portée de l'amorce est assez courte : elle ne dépasse pas le cadre de l'épisode, la résolution ayant lieu lors du cliffhanger ${ }^{9}$. À l'inverse, dans les exemples à venir, la portée de l'amorce dépassera le cadre de l'épisode.

L'hybris permet de faire peser un danger sur un personnage. Mais, ce danger n'est pas nécessairement tragique et il peut prendre plusieurs formes, comique parfois, comme dans l'une des intrigues de la saison 2 de Melrose Place. Dans l'épisode 2.13, Michael Manccini ayant eu un grave accident, Jane, son ex-femme, accepte de l'héberger. Michael lui promet : "You won't regret this Jane, I promise ${ }^{10}$. " Et, quatre épisodes plus tard ${ }^{11}$, Jane le met à la porte avec fracas, ayant largement matière à regretter de l'avoir hébergé. Cette résolution se produit à la minute vingt-sept de l'épisode, dans le début de l'acte trois, non lors du cliffhanger de l'épisode, ni même lors d'un cliffhanger de fin d'acte ${ }^{12}$. Toutes les configurations sont possibles (la résolution de l'amorce pouvant avoir lieu à tout moment) mais il est fructueux que l'amorce ait lieu lors d'un cliffhanger : cela permet de se projeter dans la suite de la fiction et d'entretenir les questionnements métadiégétiques sur lesquels repose le suspense. Ainsi, à la fin de la saison 4 de The $L$ World, alors que Bette vient de reconquérir l'amour de Jodi, cette dernière lui confie : "You'd better not break my heart. " Cette phrase est la dernière de la saison 4 : elle sert donc de cliffhanger à la saison, ce qui lui confère un poids considérable. Il semble ainsi fort probable que, par la suite, Bette brisera le cœur de Jodi, même si rien n'est moins certain.

Après ces quelques exemples d'amorces construites sur l'hybris, il est nécessaire d'énoncer les fonctions de l'amorce, sur lesquelles nous reviendrons en détail dans la suite de cet article :

1 - La fonction première de l'amorce, qui lui est inhérente, est d'introduire un événement et de l'amener de façon à ce qu'il ne semble

${ }^{8}$ Genette définit la portée en ces termes : « une anachronie peut se porter, dans le passé ou dans l'avenir, plus ou moins loin du moment "présent » [...] : nous appellerons portée de l'anachronie cette distance temporelle. » in Figures III, p.89.

${ }^{9}$ Le cliffhanger est le moment de suspense mais, dans le cadre d'une réception sérielle, il s'agit également d'un moment de frustration puisque la fiction s'interrompt. Préparer le cliffhanger par une amorce est peut-être un moyen d'atténuer la frustration inhérente au cliffhanger et de laisser le spectateur sur une satisfaction, celle d'avoir pu anticiper. ${ }_{10}$ Melrose Place, épisode 2.13, minute 27: «Tu ne le regretteras pas, Jane: je te le promets. "

${ }_{11}^{1}$ Ibid., épisode 2.18, minute 27.

${ }_{12}$ Sur les chaînes du network américain, les feuilletons sont entrecoupés de quatre pauses publicitaires, si bien que la structure en cinq actes est très apparente. Les scénaristes écrivent donc en fonction des pauses publicitaires et ménagent un cliffhanger pour chaque interruption. Ce n'est pas du tout le cas en France, car le moment de la pause publicitaire n'est jamais déterminé à l'avance. 
pas «parachuté ».

2 - L'amorce mène le spectateur à craindre (ou espérer) tel événement pour tel personnage en posant indirectement la question « que va-t-il advenir de lui ? » Elle crée donc du suspense, une attente. Aussi le procédé de l'amorce fonctionne-t-il en trois temps : amorce, attente, résolution ${ }^{13}$.

3 - L'amorce crée, insidieusement, le désir que l'événement amorcé se produise. Il s'agit probablement de la fonction la plus importante de l'amorce : créer et orienter les désirs du spectateur, comme le souligne Umberto Eco, dans Eloge de $M C$, à propos de Dantès :

Il suffit de se reporter aux trois premiers chapitres et de voir combien de fois Edmond Dantès clame à qui veut l'entendre qu'il a l'intention de se marier et qu'il est heureux : quatorze ans $\mathrm{au}$ fond du château d'If, c'est encore trop peu pour un pleurnichard de cet acabit ${ }^{14}$.

À force de dire qu'il est heureux, Dantès « énerve » le lecteur, qui finit par souhaiter que les choses ne se passent pas aussi facilement. Dans cet exemple, Dantès clame son bonheur à plusieurs reprises : il y a donc plusieurs amorces qui sont enchaînées avant la résolution. Et c'est pourquoi nous avons décidé de les appeler " amorces filées ».

4 - L'amorce accroche le spectateur à la fiction en lui faisant espérer que ses «prévisions » se réaliseront et en lui faisant attendre une « confirmation ${ }^{15}$ » de ses prévisions.

5 - L'amorce permet au spectateur de « savourer ${ }^{16}$ » par avance.

6 - La résolution de l'amorce permet de flatter l'égo du spectateur car, ayant anticipé l'événement, lorsque celui-ci se produira, le spectateur aura la satisfaction de pouvoir se dire «j'en étais sûr, je le savais ». Il éprouve ainsi le plaisir de se sentir "plus fort » que la fiction, "audessus » de la fiction - ce qui est somme toute logique puisque le spectateur se trouve dans une attitude métadiégétique, consciemment ou inconsciemment. L'amorce apporte donc au spectateur une sensation de contrôle.

7 - La résolution de l'amorce procure le plaisir de l'attente comblée.

C'est dans les amorces filées que les fonctions trois à sept de l'amorce sont les plus effectives et que la manipulation du spectateur est la plus manifeste.

${ }^{13}$ La résolution en elle-même est un événement : ainsi, l'accident de voiture de Vadim, la chute de Bran, le renvoi de Michael ou la rupture amoureuse entre Bette et Jodi.

${ }_{14}$ Umberto Eco, De Superman au surhomme, Paris, Grasset, 1993, p. 89.

${ }_{15}$ Nous empruntons les termes utilisés par Umberto Eco dans Lector in fabula, Paris, Grasset, 1985, p. 145: "Une fois qu'il [le lecteur] aura lu, il se rendra compte si te texte a confirmé ou non sa prévision. Les états de la fabula confirment ou infirment (vérifient ou falsifient) la portion de fabula anticipée par le lecteur. »

${ }^{16}$ Nous empruntons le terme utilisé par Umberto Eco dans «Eloge de Monte-Cristo » : « les multiples dévoilements de Monte-Cristo à ses ennemis, qui le public attend et savoure dès la moitié du roman ", in De Superman au surhomme, p. 30. 


\section{Créer le désir que deux personnages vivent une histoire d'amour par des amorces filées construites sur des clichés narratifs}

Les amorces filées qui contribuent à créer le désir que deux personnages vivent une histoire d'amour sont le plus souvent construites sur un cliché narratif. Le cliché met le spectateur face à un élément déjà connu, auquel il a eu affaire dans un autre feuilleton et dont on lui a déjà représenté les conséquences - en l'occurrence, ici, l'union des deux personnages. Selon Ruth Amossy et Anne Herschberg Pierrot, chaque cliché autorise "une certaine mesure de prévisibilité ${ }^{17} »$. Il est donc permis de considérer qu'une amorce peut se construire autour d'un cliché narratif. Ce que nous entendons par cliché narratif correspond aux éléments de la classe des «stéréotypes thématico-narratifs ${ }^{18} »$. Nous y ajoutons ce que Bernard Timberg appelle "stylized expressions " 19 , que nous traduirons librement par " macro expressions » et qui sont proches des « têtes d'expression » de Louis-Leopold Boilly, où le peintre amplifie la réaction émotionnelle sur la physionomie du personnage. Le terme de « macro expressions » nous paraît également faire écho aux sciences comportementales qui étudient les micro expressions : puisque, ce dont il s'agit ici, est bien d'amplifier ces micro expressions pour les rendre signifiantes dramaturgiquement. Les macro expressions sont si constitutives du soap opera et du feuilleton en général qu'il en est question dans la série Friends (NBC, 1994-2004) où le personnage de Joey, qui est lui-même comédien de soap opera, donne un cours sur le jeu de l'acteur de soap. Il declare un jour à ses étudiants : " Let's say I wanna convey that I've just done something evil. That would be your basic 'I've got a fish hook in my eyebrow and I like it. ${ }^{20}$ » - et il exécute la mimique en même temps qu'il la décrit ${ }^{21}$ (voir figure 1) :

\footnotetext{
${ }^{17}$ Ruth Amosy et Anne Herschberg Pierrot, Stéréotypes et clichés, Paris, Armand Colin, 1997, p. 75 .

${ }_{18}$ Ibid., p. 74 .

19 Hayward cite Bernard Timberg : «stylized expressions of pity, jealousy, rage, selfdoubt », p. 156 .

${ }_{20}$ Friends, épisode 3.7 : «Imaginons que je veuille faire comprendre que j’ai fait quelque chose de mal, ce serait la situation classique du 'j’ai un hameçon coincé dans un sourcil et j'aime ça.' »

${ }^{21}$ Scène disponible à l'adresse suivante :

http://www.youtube.com/watch?v=wsVqP4_kATo, lien consulté le 11 décembre 2014.
} 


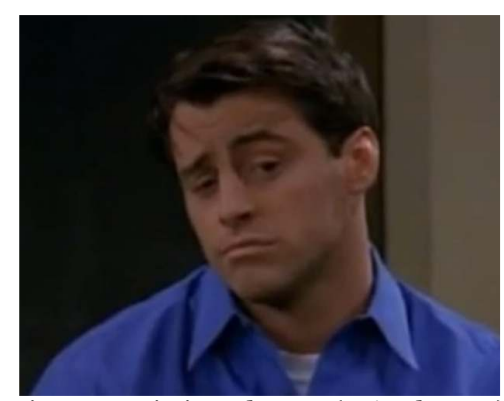

Fig. 1 : La mimique de Joey (Friends, 3.07)

Joey emploie le verbe « convey » : il s'agit donc de «transmettre » une information au spectateur, de lui faire connaître les pensées et les intentions du personnage par une mimique signifiante. Il est à noter que ces macro expressions sont également présentes dans les romansfeuilletons du XIX ${ }^{\mathrm{e}}$ siècle $^{22}$.

Dans cette partie, nous tenterons de comprendre comment les amorces filées créent et orientent les désirs du spectateur. Pour commencer, nous allons présenter les neuf amorces qui mènent à l'union d'Alison et Billy dans la saison 1 de Melrose Place. Afin de prouver qu'il est bien question de clichés fréquemment employés dans les feuilletons, nous nous référerons également à une autre relation amoureuse, celle d'Annie et Sam dans la version américaine de Life on Mars. Par souci de clarté, notre présentation suivra l'ordre chronologique de la relation d'Alison et Billy.

\section{Amorce $n^{\circ} 1$ : le sourire}

Le premier signe annonciateur d'une relation amoureuse est généralement un sourire, c'est-à-dire une macro expression. C'est le cas dans la relation d'Alison et Billy. Ils sont colocataires - Billy vient tout juste d'emménager - et, lorsqu'au début de l'épisode pilote, Alison aperçoit Billy torse nu, son sourire gêné fait comprendre qu'elle n'est pas insensible à ce qu'elle voit ${ }^{23}$ (voir figure 2). Les scénaristes américains désignent d'ailleurs ce type de situation dramatique par le

${ }^{22}$ Umberto Eco dit du Comte de Monte-Cristo : « le roman est mécanique et gauche dans la description des sentiments : ses personnages frémissent ou pâlissent, ou essuient de grosses gouttes de sueur coulant de leur front [...] ou se lèvent brusquement d'une chaise pour y retomber aussitôt... ", in De Superman au surhomme, p. 86. En effet, les termes " frémir », " tressaillir », " rougir » et "pâlir » sont extrêmement fréquents dans le texte. Le lexème «sourire » est un peu moins fréquent puisque nous ne croisons pas plus d'un sourire toutes les dix pages. Le lexème "soupir » est également présent, en moindre quantité. Enfin, le lexème "sourcil » (général au pluriel et accompagné de l'adjectif « froncés ") est le moins fréquent de tous ceux que nous avons cités.

${ }^{23}$ Melrose Place, épisode pilote, minute 21. 
terme «meet cute 24 » (« la rencontre du beau gosse »). La première amorce a pour effet d'éveiller la curiosité du spectateur.

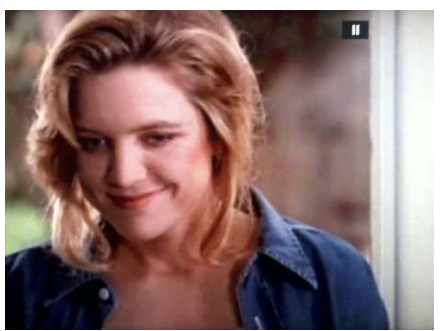

Fig. 2 : Un meet-cute?

(Melrose Place, 1.01)

S'agissant de la relation d'Annie et Sam, le sourire qu'ils échangent n'a pas lieu dans les premiers moments de leur relation mais seulement lors de l'épisode 1.6 ; cependant il s'agit bien de la première amorce de leur relation, et donc d'un meet cute. Par ailleurs, c'est lors du cliffhanger de l'épisode qu'Annie et Sam échangent ce sourire, ce qui lui confère un poids supplémentaire (voir figure 3) :
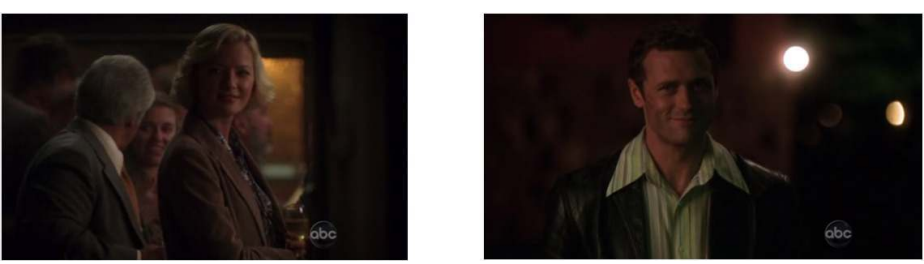

Fig. 3 : Le « meet cute » entre Annie et Sam (Melrose Place, 1.06)

\section{Amorce $n^{\circ} 2$ : la confidence}

La confidence est une étape incontournable puisque c'est par son entremise que les personnages accèdent à un certain degré d'intimité ${ }^{25}$. Dans l'épisode pilote de Melrose Place, Billy, bien qu'embarrassé, avoue à Alison qu'il ne gagne pas sa vie en tant qu'écrivain mais qu'il donne des cours de danse ${ }^{26}$. Dans Life on Mars, Annie avoue à Sam

24 " A meet-cute is a scene in film, television, etc. in which a future romantic couple meets for the first time in a way that is considered adorable, entertaining, or amusing. " http://en.wikipedia.org/wiki/Meet cute «Le meet-cute est une scène de film, de série télévisée, etc. dans laquelle un futur couple d'amoureux se rencontre pour la première fois d'une manière que l'on décrit comme mignonne, divertissante ou amusante ».

25 À noter que la confidence peut être remplacée par un point commun insoupçonné entre les deux personnages.

${ }^{26}$ Melrose Place, minute 38 
qu'elle n'a jamais pris l'avion et qu'elle n'est jamais sortie de l'état de New York ${ }^{27}$.

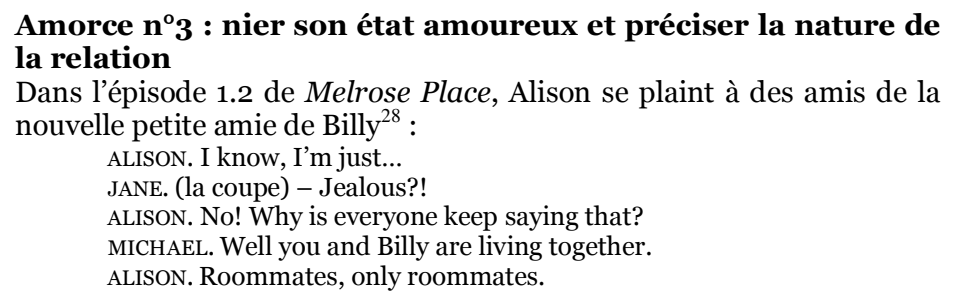

Alison nie être jalouse et précise la nature de leur relation de colocataires. Avec la troisième amorce, le système est en place et le spectateur sent que quelque chose va se passer. Dans Life on Mars, lorsque Maria tente de faire comprendre à Sam qu'il est amoureux d'Annie, Sam répond: «Annie? No, no, no, we're friends $^{29}$. » Sam nie être amoureux et précise qu'Annie et lui sont amis.

\section{Amorce $n^{\circ} 4$ : faire semblant d'être en couple}

Faire semblant d'être en couple oblige les personnages à une certaine proximité physique. Dans l'épisode 1.7 de Melrose Place, afin de se faire valoir, Billy fait croire à ses parents qu'Alison est sa petite amie ; aussi Billy et Alison sont-ils amenés à dormir dans le même lit. Dans Life on Mars, Annie et Sam étant policiers, c'est pour une infiltration qu'ils font semblant d'être en couple tout au long de l'épisode 1.14.

\section{Amorce $n^{\circ} 5$ : dans un état de conscience altéré, fantasmer sur l'autre}

Dans l'épisode 1.16 de Melrose Place, Billy prend des anti-douleurs pour apaiser une rage de dent, puis il s'endort. Le spectateur assiste à un rêve dans lequel Alison et lui s'embrassent. De même, dans Life on Mars, le spectateur voit le rêve de Sam qui a pris du LSD à son insu : il fantasme sur plusieurs femmes, dont Annie ${ }^{30}$. Ce cliché, dont ces exemples illustrent une première forme, en revêt parfois une seconde : dans un état de conscience altérée, le personnage avoue son état amoureux. Dans l'épisode 4.11 de Melrose Place, Jane, Jake et sa petite amie du moment ont bu quelques verres d'alcool, et Jane dit à Jake,

${ }^{27}$ Life on Mars, épisode 1.14 , minute 12

${ }^{28}$ Melrose Place épisode 1.2, minute 37 Alison : "Je sais, je suis juste ... Jane (la coupe) : « Jalouse ?! » Alison : "Non! Pourquoi est-ce que tout le monde n'arrête pas de dire ça ? Michael : «Ben, toi et Billy, vous vivez ensemble. » Alison : "Colocataires, on est juste colocataires. »

${ }^{29}$ Life on Mars, épisode 1.14, minute 27. « Annie ? Non, non, non, on est amis. 》

${ }^{30}$ Life on Mars, épisode 1.4, minute 29. 
devant sa petite amie : "I've always liked you Jake, you're a good guy, you're a really really decent guy. And so good looking. Isn't he good looking !? ${ }^{31}$ » Ici, l'alcool mène Jane à des compliments qui ressemblent à une déclaration d'amour. Dans la relation entre Kimberly et Michael (toujours dans la saison 4 de Melrose Place), ce cliché est poussé à l'extrême. Michael (qui est médecin), alors qu'il prétend faire une injection de vitamines à Kimberly, lui injecte du sérum de vérité car il la soupçonne de vouloir s'en prendre à lui. Kimberly, sous l'effet du sérum, lui dit qu'elle ne lui fera plus jamais de mal car elle l'aime de tout son cœur et de toute son âme ${ }^{32}$. L'état de conscience altérée engendre donc soit le fantasme soit l'aveu.

\section{Amorce $n^{\circ} 6$ : sauver l'autre d'un danger}

Sauver l'autre d'un danger équivaut au topos du prince charmant qui sauve la princesse. C'est le cas dans la saison 4 de Melrose Place où Jake sauve Jane de la noyade ${ }^{33}$ et Michael sauve Kimberly d'une explosion $^{34}$. À partir du moment où un personnage sauve l'autre, ils se retrouvent liés. Ce cliché arrive de façon un peu atténuée entre Alison et Billy: dans l'épisode 1.18, alors qu'une une tumeur a été diagnostiquée à Alison, Billy trouve l'argent nécessaire à l'intervention chirurgicale (ce qui constitue un vrai défi) et soutient Alison de ses encouragements constants avant et après l'opération. Plus ostensiblement dans Life on Mars, Sam sauve Annie des mains d'un tueur au cours de l'épisode 1.7 .

\section{Amorce $n^{\circ} 7$ : nier son état amoureux (bis)}

Chacun des deux personnages se doit de nier son état amoureux. Dans l'épisode 1.18 de Melrose Place, Billy parle d'Alison à des amis communs, Jake et $\mathrm{Jo}^{35}$ :

JAKE. Billy, you know what I think: you should wonder how you really feel about Alison.

BILLY. I don't have feelings for Alison!

La négation de Billy est très affirmée et très énergique, et sonne comme une dénégation. Dans l'épisode 1.17 de Life on Mars, c'est au tour d'Annie de nier ses sentiments lors d'une dispute avec Ray, arbitrée par

\footnotetext{
${ }^{31}$ Melrose Place, épisode 4.11, minute 23. "Je t’ai toujours apprécié, Jake. T'es un mec bien. T'es un mec vraiment honnête, franchement. Et puis, super beau. (S’adressant à la petite amie de Jake) N'est-ce pas qu'il est beau !? »

${ }^{22}$ Melrose Place, Épisode 4.19, minute 28. Notons que les scénaristes ont donc de nouveau employé ce cliché huit épisodes seulement après la déclaration d'amour alcoolisée de Jane.

33 Ibid., épisode 4.18 , minute 43.

34 Ibid., épisode 4.20, minute 55. Notons qu'ici le cliché est utilisé dans deux épisodes consécutifs.

35 Ibid., épisode 1.18, minute 18. Jake : "Billy, tu devrais te demander ce que tu ressens vraiment pour Alison. » Billy : « Je ne ressens rien pour Alison! »
} 
le commissaire ${ }^{36}$ :

RAY. Ah, please! They're in love! Tyler [Sam] burps and she looks at him like he's singing a melody. To me the all thing is nausia!

LE COMMISSAIRE. All right, all right. Is that true Norris [Annie]?

ANNIE. No sir.

\section{Amorce ${ }^{\circ} 8$ : hybris}

L'hybris étant un stéréotype thématique, nous nous permettons de l'inclure à cette liste d'amorces. On trouve l'hybris sous forme de conseil dans l'épisode 1.20 de Melrose Place. Jo, voisine et amie d'Alison, lui dit : "Alison, some advise : never go out with anyone in the building ${ }^{37}$ ». Il n'y a pas d'exemple similaire dans Life on Mars.

\section{Amorce ${ }^{\circ} 9$ : la femme panse les plaies de l'homme blessé}

Il faut évoquer un cliché qui, bien qu'absent de la relation d'Alison et Billy, est fort courant. Il s'agit du personnage, quasi christique, de la femme qui panse les plaies de l'homme, au sens littéral uniquement, et qui implique une proximité physique. Ce cliché est présent à la minute 12 de l'épisode 1.9 de Life on Mars. On le trouve dans de nombreux feuilletons ainsi que dans certains films.

\section{Amorce $n^{\circ} 10$ : dernier retardement}

Le dernier retardement est une étape presque obligatoire. Dans Melrose Place, Billy se met en couple avec Amanda depuis l'épisode 1.23 jusqu'à l'épisode 1.24 ; l'union d'Alison et Billy semble donc compromise. Dans l'épisode 1.16 de Life on Mars, Sam tente de partir $^{38}$, ce qui équivaudrait à ne plus jamais revoir Annie.

Revenons maintenant sur les fonctions 3 et 4 de l'amorce filée. Il est donné au spectateur de nombreuses amorces, le spectateur les remarque, consciemment ou inconsciemment (selon sa " compétence narrative ») et présume qu'un événement va se produire - en l'occurrence l'union des couples Alison/Billy et Annie/Sam. Quelque soit le nombre d'amorces qui compose l'amorce filée, le spectateur ne peut qu'émettre des hypothèses, car l'amorce n'apporte jamais de certitude. Dans Lector in fabula, Umberto Eco écrit: « le lecteur, en faisant ces prévisions, assume une attitude proportionnelle (il croit, il

${ }^{36}$ Life on Mars, episode 1.17, minute 21. Ray : «Ils s'aiment ! Tyler [Sam] rote et elle le regarde comme s'il chantait une chanson. Moi je trouve ça écoeurant. » Le commissaire : "Ça va, ça va. Est-ce que c'est vrai Norris [Annie] ? " Annie : " Non monsieur. »

37 Melrose Place, épisode 1.20, minute 25. Jo: «Alison, un conseil : ne sors jamais avec quelqu'un de la résidence. »

${ }^{38}$ La diégèse se déroule en 1973 . Sam, un homme qui vient du futur (on ne sait comment), est bloqué en 1973 depuis l'épisode pilote. Dans l'épisode 1.16, Sam, se croyant prisonnier d'un rêve, est prêt à se suicider pour y mettre un terme et rentrer chez lui. 
désire, il souhaite, il espère, il pense) quant à l'évolution des choses ${ }^{39}$. » Les possibilités diégétiques sont donc ouvertes et le spectateur est tiraillé entre deux possibilités : l'événement peut se produire ou non. Dans les feuilletons, le spectateur est amené à faire des prévisions sur la suite de la diégèse et devient, en quelque sorte, un enquêteur métadiégétique; or, il est humain d'espérer être un bon enquêteur plutôt qu'un mauvais. C'est ici qu'opère la troisième fonction de l'amorce - celle de créer et d'orienter les désirs du spectateur. Car, après avoir remarqué une ou plusieurs amorces, le spectateur finit par espérer qu'il a su décoder la fiction, au point d'espérer que ses prévisions se vérifient. L'espoir d'être un bon enquêteur étant plus de l'ordre de l'inconscient que du conscient et les prévisions de l'ordre de "l'intime conviction ", c'est très sincèrement que le spectateur désire l'union des couples Alison/Billy et Annie/Sam - et, peut-être, sans vraiment savoir pourquoi. L'acte est manipulateur car le spectateur croit que ce désir provient de lui, alors que tout cela lui a été savamment insufflé par les créateurs.

De plus, à partir du moment où le spectateur est mu par l'espoir qu'un événement précis advienne, il est légitime de penser qu'il continue de suivre la fiction au moins en partie parce qu'il attend que cet événement se produise, parce qu'il attend d'avoir confirmation que ses prévisions étaient justes. Il s'agit de la quatrième fonction de l'amorce filée : accrocher le spectateur à la fiction où il espère trouver la preuve qu'il est un bon enquêteur métadiégétique. Même si l'accumulation des amorces porte à croire que ces couples finiront par se former, rien non plus ne l'assure. Les propos d'Umberto Eco quant aux redondances dans Le Comte de Monte-Cristo peuvent s'appliquer aux amorces filées :

On découvre que les horribles intempérances stylistiques [les redondances] sont, certes, des «chevilles », mais qu'elles ont une valeur structurale, comme les barres de graphite dans les réacteurs nucléaires, ralentissant le rythme pour rendre nos attentes plus lancinantes, nos prévisions plus hasardeuses $4^{\circ}$.

Chaque amorce est une pique qui constitue l'effet lancinant - chaque amorce est une partie visible du fil blanc dont est cousue l'histoire - et certaines amorces rendent « nos prévisions plus hasardeuses », comme l'amorce $\mathrm{n}^{\circ} 10$ (le dernier retardement).

Bien qu'il soit évident, que l'événement amorcé se produira, le spectateur est maintenu dans un état de doute. Certes, ce doute est « mince » mais il est justement calibré pour titiller le spectateur et lui faire convoiter une confirmation. Les amorces filées créent, puis attisent des désirs que le spectateur croit siens, et accrochent ce dernier

39 Eco, Lector in fabula, p. 145

$4^{40}$ Eco, De Superman au surhomme, p. 101. 
à la fiction en lui faisant guetter le moment où ses prévisions se réaliseront. Le procédé est donc doublement fructueux et " manipulateur » par essence.

En ce qui concerne les relations amoureuses, ce procédé peut s'étendre sur une longue période : neuf mois ${ }^{41}$ pour Alison et Billy, cinq mois $^{42}$ pour Annie et Tyler. La confirmation sait se faire attendre ${ }^{43}$. Or, l'attente est un élément primordial du feuilleton ${ }^{44}$. Billy et Alison s'embrassent enfin dans l'épisode 1.28, et Annie et Sam dans l'épisode 1.

\section{Créer le désir qu'un personnage soit le « méchant »}

Le personnage du méchant est très fréquent dans les feuilletons. Le plus souvent, il est immédiatement identifié par des signes explicites mais, parfois, il l'est par des amorces, c'est-à-dire par des signes implicites. Nous illustrerons ce propos en analysant les amorces qui, dans les épisodes 1.7 et 1.8 de Dexter, mènent le spectateur à souhaiter que Rudy, petit ami de la sœur du héros, soit le méchant.

De nombreux éléments, plus souvent métadiégétiques (hybris, cliché, émotion stéréotypée) que diégétiques, vont mener le spectateur à penser que Rudy est le tueur - mais, de son côté, Dexter n'aura accès à aucun de ces éléments d'information et restera dans l'ignorance jusqu'au dernier moment. Umberto Eco définit cette « reconnaissance contrefaite » en ces termes :

Avec la reconnaissance contrefaite, le personnage tombe des nues lors de la révélation, tandis que le lecteur sait déjà ce qui se passe. Exemple type: les multiples dévoilements de MonteCristo à ses ennemis, que le public attend et savoure dès la moitié du roman ${ }^{45}$.

Par le biais des amorces, non seulement le public attend (quatrième fonction de l'amorce), mais il savoure aussi à l'avance le moment du dévoilement (cinquième fonction). De même, Jennifer Hayward insiste sur la notion de plaisir :

Soap viewers, far from being 'tricked' into expecting a climax that never arrives, profoundly enjoy the extended suspense, which has been refined to an art over centuries of

\footnotetext{
${ }^{41}$ Neuf mois de diffusion se sont écoulés entre la première amorce et la résolution, c'est-àdire entre l'épisode pilote (diffusé le 8 juillet 1992) et l'épisode 1.28 (diffusé le 7 avril 1993). ${ }^{42}$ Cinq mois de diffusion (quatre mois et demi pour être exact) se sont écoulés entre l'épisode 1.6 (diffusé le 13 novembre 2008) et l'épisode 1.17 (diffusé le $1^{\mathrm{er}}$ avril 2009).

43 Â ce titre, dans Plus belle la vie, la relations de Samia Nassri et Jean-Paul Boher, dont les amorces reposent sur sept des clichés narratifs que nous avons évoqués ici (à l'exception des amorces que nous avons numérotées 1,5 et 9), s'étend sur une période de deux ans. ${ }_{44} \mathrm{Cf}$. la célèbre phrase de Wilkie Collins : « Make them laugh, make them cry, make them wait ».

45 Eco, De Superman au surhomme, p. 30.
} 
serialization ${ }^{46}$.

Le moment dont parle Jennifer Hayward, moment de suspense étendu très apprécié des spectateurs, correspond au moment dont parle aussi Umberto Eco, où le public « savoure » par avance. En ce qui concerne l'amorce filée, ce moment commence avec la première amorce et se termine avec la résolution.

Précisons une particularité de l'intrigue de Dexter: le spectateur, en visionnant l'épisode 7 , a plus ou moins oublié le personnage de Rudy qui n'a fait qu'une brève apparition dans l'épisode $1.5^{47}$, et les scénaristes, avant de créer le désir que Rudy soit le tueur, vont d'abord créer celui que Neil Perry, individu présumé coupable par la police, ne le soit pas. Résumons le début de la première saison de Dexter. Le commissariat central de Miami est sur la piste d'un tueur en série nommé « the ice truck killer » (« le tueur au camion réfrigéré »). Dexter, le héros, qui travaille au département scientifique du commissariat, en partie pour mieux cacher qu'il est lui même tueur en série, a été contacté par «the ice truck killer» et, sans qu'ils ne se soient jamais vus, un jeu, puis un respect mutuel, s'instaurent entre les deux hommes. Dexter admire les techniques sophistiquées de cet autre tueur et voit en lui la personne qui, enfin, pourrait le comprendre et l'accepter tel qu'il est. Dans l'épisode 7, tous les membres de l'équipe de police sont persuadés de la culpabilité du suspect numéro un, un homme du nom de Neil Perry. Dexter craint donc que son «ami » ait été pris et ne le dénonce. Mais, en voyant la photo de Neil Perry, Dexter et le spectateur découvrent un visage, et surtout une expression, en totale contradiction avec ce que l'on est en droit d'attendre de la part de quelqu'un que l'on admire (voir figure 4) :

${ }^{46}$ « Les spectateurs de soap, loin d'être trompés par l'attente d'un paroxysme qui n'arrive jamais, trouvent un grand plaisir dans le prolongement du suspense, lequel est devenu un véritable art au bout de deux siècles de sérialité. " Jennifer Hayward, Consuming Pleasures, Lexington, The University Press of Kentucky, 1997, p. 151. De même, Ruth Amossy et Anne Herschberg Pierrot considèrent que «les stéréotypes sont sources de suspense et de plaisir ", op. cit., p. 81 .

47 Genette, Figures III, p. 112, cite comme exemple d'amorce: «par exemple faire apparaître dès le début un personnage qui n’interviendra vraiment que beaucoup plus tard ». 


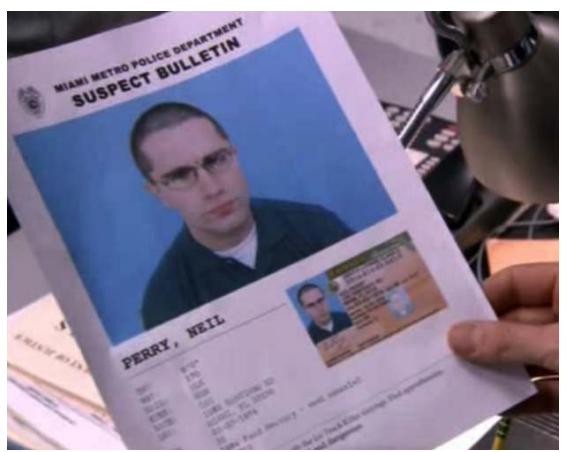

Fig. 4 : Découverte de Neil Perry (Dexter, 1.07)

Dexter demande à sa sœur Deb, qui fait partie de l'équipe de police: "You really think this is the guy!? Hardly look like a guy capable of terrorising Miami, a science camp maybe ${ }^{48}$. » Ce jugement ironique et méprisant de Dexter - et drôle, ce qui désacralise d'autant plus le suspect - est fondé sur l'apparence physique. Or, l'apparence physique n'est pas une preuve, ni même un indice. Il s'agit d'une amorce sous forme d'enthymème («the ice truck killer» est sophistiqué, Neil Perry semble frustre, il est donc peu probable que Neil Perry soit « the ice truck killer »). Cela engage le spectateur dans une attitude métadiégètique, qui le conduit à espérer, pour le bien de la diégèse, que le grossier personnage de la photo, Neil Perry, ne soit pas le tueur en série ardemment recherché depuis l'épisode pilote.

La deuxième amorce est dans le prolongement de la première. Elle a lieu vingt minutes plus tard. L'équipe de police fouille le domicile de Neil Perry, insalubre et empli d'objets hétéroclites plutôt kitsch, parmi lesquels on trouve de nombreux animaux empaillés - ce qui incite le spectateur à souhaiter que Neil Perry ne soit pas « the ice truck killer » car son domicile ne correspond pas à l'image sophistiquée que Dexter s'est faite du tueur. Aussi Dexter, avec qui le spectateur est donc en accord, assène-t-il à sa sœur : «I hate to break this to you, Deb, but you know you got the wrong guy here ${ }^{49}$. » Une troisième amorce, cinq minutes plus tard, réitère la thèse du contraste entre l'intelligence supposée du tueur et l'aspect frustre de Neil Perry. Dexter pense en voix off: "The first time I saw his work I felt like a Spanish explorer landing on the shores of a new world; no blood in the victims, no spatter, no stain; Deb is wrong about him ${ }^{50}$." Puis Dexter, et le

${ }^{48}$ Dexter, coffret DVD Showtime Entertainment et Paramount Pictures (2007), épisode 1.7, minute 9 .

49 Ibid., minute 20.

${ }^{50}$ Ibid., minute 25. 
spectateur avec lui, commence à avoir des doutes et à envisager la culpabilité de Neil Perry qui, lors de son interrogatoire (interrogatoire retransmis par vidéo - si bien que Dexter voit Neil Perry sans être vu de lui) est un peu plus raffiné que ce à quoi l'on s'attendait. Dexter doute. En observant le visage de Neil Perry sur l'écran vidéo, il confie à la caméra : " At least I hope he's a fraud ${ }^{51}$. » Cette quatrième amorce a pour fonction de rendre "nos prévisions plus hasardeuses". Il s'agit donc moins d'une amorce que d'un leurre ${ }^{52}$. Mais, dès le plan suivant, le spectateur est aiguillé sur la piste de Rudy. Voici les deux plans successifs $^{53}$ :
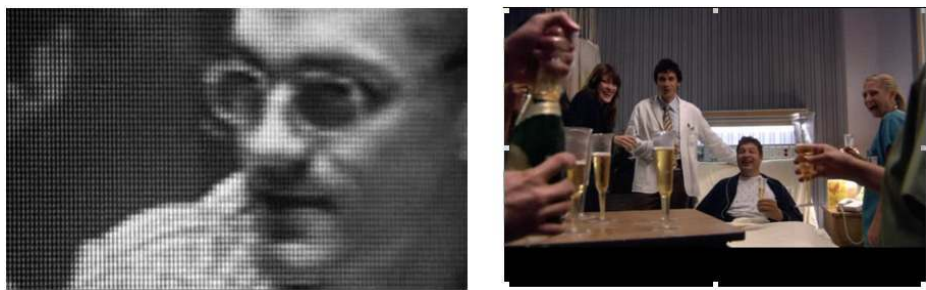

Fig. 5 : Plans successifs de Neil Perry à Rudy (Dexter, 1.07)

Après un gros plan sur Neil Perry, que le spectateur et Dexter espèrent être un imposteur, vient un plan large dans lequel Rudy se trouve au centre, comme au bout d'un tunnel. Or Rudy, qui avait fait une brève apparition dans l'épisode 1.5, est médecin spécialisé en membres artificiels, ce qui fait de lui un suspect potentiel, " the ice truck killer » ayant la particularité de démembrer ses victimes. Rudy est de surcroît bien plus séduisant et raffiné que Neil Perry. Cette cinquième amorce repose sur la succession de deux plans, c'est-à-dire sur un effet de montage. Le fondement de l'amorce est donc différent de celui de l'indice des romans policiers, qui repose sur des éléments de la diégèse ${ }^{54}$, alors que l'amorce repose sur des éléments métadiégétiques ;

${ }^{51}$ Ibid., minute 37.

${ }^{2}$ Genette définit le leurre ainsi: «C'est d'ailleurs sur cette compétence même [la compétence narrative du lecteur] que se fonde l'auteur pour tromper le lecteur en lui proposant parfois de fausses, ou leurres ", in Figures III, p. 114.

53 Dexter, épisode 1.7, plan 1 de la minute 37:40 à 37:45, plan 2 de 37:45 à 37:49.

54 Permettons-nous une comparaison avec le roman policier. Disons que la diégèse d'un roman policier est une "équation à une inconnue ", que le lecteur, parallèlement au détective de la fiction, cherche à résoudre. Il est reconnu par la critique que le roman policier nécessite un récepteur « actif». En revanche, on ne peut pas vraiment dire que la diégèse d'un feuilleton soit une "équation à une inconnue ». Dans Monte-Cristo par exemple, il est évident dès le premier chapitre que Dantès est le héros, et à partir du moment où l'Abbé Faria complète l'équation du titre du roman en disant à Dantès qu'il y a un trésor sur l'île de Monte-Cristo, il est évident que Dantès trouvera le trésor (car le personnage dont le roman porte le nom pour titre ne saurait être autre que le héros), et se vengera ensuite (il ne saurait en être autrement selon l'idéologie de la consolation émise par 
on pourrait dire que l'amorce est un indice métadiégétique.

Une sixième amorce, sous forme de cliché narratif, vient tout de suite renforcer l'effet de la cinquième qui, certes, était ténu. Il faut savoir que Deb, la sœur de Dexter, que l'on peut voir à gauche de Rudy sur les images ci-dessus et ci-dessous, a été présentée depuis le début de la série comme le personnage dont les histoires sentimentales s'avèrent toujours catastrophiques - elle représente la jeune fille qui ne cesse de faire de mauvais choix amoureux (« who falls for the wrong guy »). Or, Deb semble sous le charme de Rudy, comme on peut le voir dans l'image suivante ${ }^{55}$ :

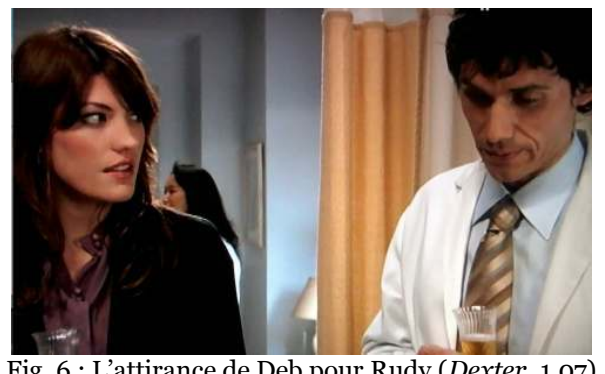

À la fin de la scène, Deb accepte d'aller dîner avec Rudy en tête-à-tête. Aux yeux du spectateur, Rudy devient alors un suspect idéal car, en plus d'avoir les connaissances médicales nécessaires et en plus d'être plutôt raffiné et séduisant, il met potentiellement Deb en danger de par leur nouvelle proximité sentimentale, ce qui n'est pas le cas de Neil Perry, et ce qui fait de Rudy un méchant bien plus intéressant. Pourtant, cinq minutes plus tard ${ }^{56}$, la septième amorce sème le doute (il s'agit donc d'un leurre) : Neil Perry, dont l'interrogatoire se poursuit, revendique son crime et, pour donner foi à ses propos, explique les procédés techniques utilisés avec une précision qui convainc l'assemblée (y compris Dexter) de sa culpabilité. Le spectateur, bien qu'engagé sur la piste de Rudy, est maintenu dans un état de doute. Cinq autres minutes plus tard ${ }^{57}$, lorsque Deb et Rudy s'embrassent (ce qui constitue une amorce sous forme de cliché

Umberto Eco dans De Superman au surhomme : si Dantès n'obtenait pas vengeance, ce ne serait pas «juste » et l'équilibre du quasi-monde ne serait pas rétabli). Le feuilleton ne nécessite donc pas un récepteur particulièrement actif ; mais il encourage l'activité de son récepteur en portant l'attention de ce dernier sur l'énonciation et sur les procédés d'anticipation. Selon nous, le feuilleton engage donc davantage son récepteur dans un rôle d'enquêteur de la narration.

55 Dexter, épisode 1.7, minute 37.

${ }^{5}$ Ibid., minute 42.

${ }^{7}$ Ibid., minute 46 . 
narratif), le spectateur est de nouveau orienté sur la piste de Rudy. Il est donc ballotté d'une piste à l'autre. Mais, malgré tout, il en sait plus que Dexter qui, lui, n'a jamais été orienté sur la piste de Rudy.

Enfin, lors du cliffhanger de l'épisode, Dexter et le spectateur ont la confirmation que Neil Perry n'est pas "the ice truck killer». Dexter, qui n'a jamais vu Neil Perry que par écran vidéo interposé, trouve l'occasion de se faufiler dans la salle d'interrogatoire. Précisons que si Dexter ne connaît pas le visage du tueur, ce dernier connaît le sien. Or, Neil Perry, lorsqu'il se retrouve face à Dexter, lui demande : "Who the fuck are you ! $?^{58}$ » sur un ton méprisant qui ne saurait provenir de la personne avec qui Dexter entretient une relation de respect. Cette confirmation est soulignée par un changement de musique, passant d'une atmosphère tendue à une atmosphère joyeuse, voire victorieuse (la scène est presque comique), et par le changement de l'expression faciale de Dexter qui clôt l'épisode en passant d'un visage sombre à un sourire de contentement.

La confirmation que Neil Perry n'est pas le tueur étant un moment dramatique fort, il est cohérent qu'elle advienne lors du cliffhanger, qui est le point culminant - de plus, elle atténue la frustration inhérente à ce moment de séparation d'avec la fiction. Cette confirmation comble l'attente provoquée par l'amorce et apporte au spectateur une sensation de contrôle par la satisfaction d'avoir su anticiper (combler et flatter sont à notre sens deux plaisirs distincts qui ici se cumulent). En cela, l'amorce est un élément structurel, constitutif de ce qu'Umberto Eco, en parlant de "la dynamique sollicitationsolution ${ }^{59}$ " des romans populaires, appelle une «machine gratifiante ». La métaphore de la " machine gratifiante » nous semble particulièrement appropriée pour décrire Dexter car, après avoir eu la confirmation que Neil Perry était un imposteur, le spectateur attend de savoir que Rudy est bien « the ice truck killer ».

Dans l'épisode 1.8, quatre amorces viennent titiller le spectateur avant qu'il n'obtienne cette dernière confirmation lors du cliffhanger. Tout d'abord, à la suite de leur premiers ébats amoureux, Rudy dit à Deb : " I love your legs, I love your arms, I couldn't have made them better myself ${ }^{60}$.» Rudy parle de jambes et de bras dans des circonstances où l'on s'attend à l'évocation de parties du corps plus spécifiquement féminines - cette amorce repose donc sur un cliché non conforme - et sa comparaison implicite avec son travail dénote une certaine mégalomanie. Puis, plus tard dans l'épisode, le spectateur partage le malaise de Deb lorsque Rudy lui demande s'il peut réaliser un moulage de ses jambes afin de fabriquer une prothèse pour l'une de

${ }^{8}$ Ibid., minute 51. « Putain, vous êtes qui vous ?! »

59 Eco, De Superman au surhomme, p. 22.

${ }^{60}$ Dexter, episode 1.8, minute 19. " J'aime ton corps, j'aime tes jambes, j'aime tes bras. Je n'aurais pas su faire mieux. » 
ses patientes : «I wanna give her a smoking new pair of legs. Yours. ${ }^{61}$ » Dans cette même scène, le sourire malicieux de Rudy, filmé de surcroît en contre-plongée, constitue une amorce construite sur une macro expression : celle du sourire, non plus amoureux comme pour le meet cute, mais cette fois malicieux (voir figure 7) ${ }^{62}$.

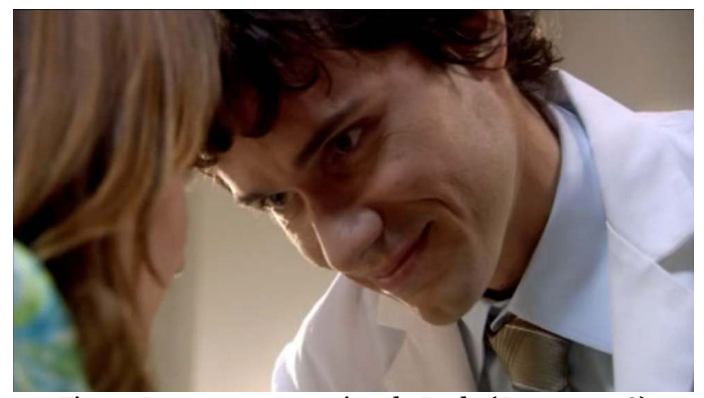

Fig. 7 : La macro expression de Rudy (Dexter, 1.08)

Une dernière amorce est présente dans la scène finale de l'épisode (elle précède la confirmation de peu). Deb et Rudy, chacun chez soi, se parlent au téléphone ${ }^{63}$ :

RUDY. And, I'm a neat freak.

DEB. You're just full of surprises, ah?!

RUDY. Oh you have no idea!...

Le terme «monstre » (freak) est employé. Si Deb ne peut imaginer que Rudy soit un monstre, les propos sont des plus explicites pour le spectateur. La confirmation a lieu quelques secondes après. Notons que la confirmation se doit d'être absolue et ne laisser aucun doute possible. Après avoir raccroché, Rudy se dirige vers son bureau, sur lequel on découvre un exemplaire similaire de la poupée Barbie offerte à Dexter par «the ice truck killer ». Cet élément est suffisant pour incriminer Rudy mais la confirmation est loin d'être aboutie. De la poupée sur le bureau, la caméra passe à l'écran d'ordinateur ; s'y trouve le mail que Dexter à envoyé au tueur : " frozen barbie looking for Ken. Dear Ken, I'm in pieces. Why the cold shoulder? Love, Barbie ». À ce mail, Rudy répond: "Barbie, be patient. One day we'll share a cold one. ${ }^{64} » \mathrm{La}$ réponse de Rudy est l'élément le plus important de cette

${ }^{61}$ Ibid., minute 34 . «Je veux lui donner une paire de nouvelles jambes superbe. Les tiennes. »

${ }_{62}$ La macro expression du sourire malicieux est fréquemment utilisée dans les feuilletons, en particulier par le personnage de J.R. dans Dallas (CBS, 1978-91).

${ }^{6} 3$ Dexter, épisode 1.8, minute 51. Rudy : «Et je suis un monstre de propreté. » Deb : « Tu es vraiment plein de surprises, hein !? » Rudy : «Oh, tu n’as pas idée !... »

${ }^{64}$ L'échange de mails entre Dexter et le tueur : «Barbie frigorifiée cherche Ken. Cher Ken, 
confirmation (qui n'est toujours pas finie) car le spectateur a accès au sens second de la phrase "one day we'll share a cold one " quand Dexter n'aura accès qu'au sens premier. Le spectateur comprend immédiatement que l'indéfini «one » représente Deb et non un cocktail. Ainsi, le cliffhanger contient la confirmation mais active le suspense en soulignant le danger encouru par $\operatorname{Deb}^{65}$. De plus, la position de supériorité du spectateur sur les personnages, sur Dexter en l'occurrence, est réaffirmée par ce procédé typique de l'ironique dramatique ${ }^{66}$. Rudy quitte son bureau et se rend dans une pièce (voir figure 8) ; la caméra s'approche très lentement de la porte dont s'échappe une fumée qui semble provoquée par le froid, qui est une des caractéristiques de « the ice truck killer » (voir figure 9):

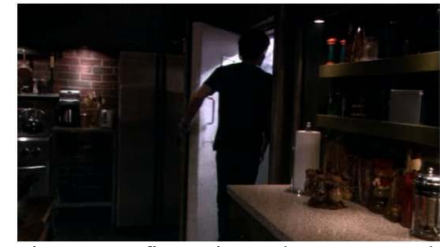

Fig. 8 : confirmation a (Dexter, 1.08)

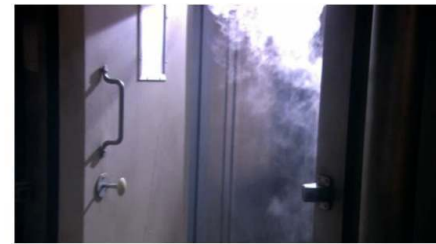

Fig. 9 : confirmation b

Toujours lentement, la caméra s'approche du seuil de la porte et l'on aperçoit des bocaux dont le contenu reste indéterminé, comme pour ralentir une confirmation qui pourtant est déjà là. Puis, la caméra entre enfin dans la pièce. Du fait de la surexposition, on discerne le premier plan avant le second. Au premier plan, des membres découpés et empaquetés à la façon de " the ice truck killer »; au second, Rudy qui a eu le temps de mettre un tablier, des lunettes de protection et ses gants. Ensuite, on voit Rudy prendre une perceuse, puis la caméra s'éloigne mais on entend le son de la perceuse. L'épisode se termine ainsi. La confirmation que Rudy est le tueur repose sur l'accumulation de cinq preuves : la poupée Barbie, le mail de Dexter, la réponse de Rudy, le froid qui s'échappe de la pièce secrète de Rudy, et Rudy s'apprêtant à découper des cadavres. Plus la confirmation a été attendue, plus elle se doit d'être magistrale.

Ce stratagème est également présent dans d'autres feuilletons : par exemple, dans l'épisode 1.22 de Melrose Place, où Jo retrouve un ami de lycée, Reed. Les amorces menant à croire que Reed est mal intentionné sont les suivantes. La première repose sur son apparence

je suis en morceaux. Pourquoi me bats-tu froid ? Bises, Barbie » « Barbie, sois patiente. Un jour, on s'en offrira un à deux, bien frais. »

${ }^{6}$ Il en était de même dans le cliffhanger de l'épisode 1.7 qui, en apportant la confirmation de l'imposture de Neil Perry, ouvrait la question « mais si ce n'est pas lui, qui est-ce? » 66 Ainsi, dans les épisodes suivants le spectateur aura plaisir à voir Dexter côtoyer Rudy sans savoir que ce dernier est le tueur qu'il recherche et avec qui il a déjà établi une relation. 
physique : Reed ressemble fortement à l'ex petit-ami de Jo, Jake, mais les traits de son visage sont un peu moins parfaits. C'est Jake en moins bien. Or, le feuilleton n'a pas besoin d'un second Jake (la place est déjà prise). La deuxième amorce est un élément diégétique : Reed dit à Jo qu'il sort de prison, qu'il a été inculpé pour trafic de stupéfiants mais qu'il était innocent. Puis une troisième amorce est mise en relief par un élément structurel, le cliffhanger de l'épisode 1.22, où Reed fait à Jo la surprise d'emménager soudainement dans la même ville qu'elle. Les amorces s'accumulent dans les épisodes suivants, jusqu'à nous montrer enfin Reed entreposer de la drogue dans la soute de son bateau dans l'épisode 1.24. De l'épisode 1.22 à l'épisode 1.24, le spectateur attend, espère, désire, " savoure " à l'avance, le fait que Reed soit le méchant, et que Jo ne le sache pas. Il en va de même du premier au dernier épisode de la saison 1 de Homeland. Le spectateur anticipe et se régale avant l'heure à l'idée que Brody, soldat américain de retour au pays après huit ans de captivité en Irak, ait été " retourné » lors de sa captivité, et qu'il incarne un danger pour le pays. Le spectateur est conduit à ce désir, d'une part, pour le bien du personnage de Carrie, agent de la CIA convaincue que Brody est passé à l'ennemi et qui prend de grands risques pour le prouver à sa hiérarchie ; d'autre part, pour le bien de la diégèse car si Brody n'est pas dangereux, la fiction n'a pas lieu d'être ${ }^{67}$.

Les amorces sont la matière première du suspense et, insidieusement, elles accrochent le spectateur à la fiction. Elles orientent les désirs du spectateur qui, inconscient ou même conscient de cette manipulation, "attend et savoure » à l'avance. Enfin, la résolution de l'amorce procure le plaisir de l'attente comblée tout en flattant le spectateur d'avoir su anticiper. Elles incitent donc le spectateur à être " actif », en lui donnant la sensation de " participer » à la fiction, ainsi que l'illusion d'un contrôle. Par ailleurs, elles sont utilisées dans trois cas : pour mettre un personnage en danger, pour orienter les relations amoureuses des personnages et pour introduire le personnage du méchant. Elles jouent donc sur les points les plus forts sur lesquels repose la dramaturgie - la mort, l'amour, le bien et le mal. Nous avons insisté sur l'aspect manipulateur des amorces, qui abusent le spectateur en créant des désirs que ce dernier croit sien tout en excitant un besoin de confirmation et de consolation, mais nous n'avons pas abordé le plaisir intellectuel qu'elles peuvent provoquer, celui de prévoir les éléments dramatiques et leurs combinaisons. Les feuilletons dont il a été ici question correspondent à ce qu'Umberto Eco appelle « la fabula à l'état pur, sans scrupule et libre de tensions

${ }^{67} \mathrm{Si}$ Brody n'était pas dangereux pour le pays, ce début d'histoire ressemblerait à une fin (le retour du héros dans son pays). 
problématiques $^{68}$ », c'est-à-dire des fictions dont la dynamique est centrée sur la dramaturgie et où chaque amorce est une " perche » que l'auteur tend au spectateur, une invitation au grand jeu de la lecture métadiégétique. Selon Umberto Eco, ce type de dramaturgie "va de Tom Jones aux Trois Mousquetaires jusqu'aux descendants actuels du roman-feuilleton » :

Ici, l'histoire, en résolvant ses propres nœuds, se console et nous console. La fin est point pour point ce que l'on attendait. [...] Mais ce faisant, il [le roman populaire] déploiera une telle énergie, il libérera un tel bonheur, sinon inventif du moins combinatoire, qu'il proposera une jouissance qu'il serait hypocrite de nier69.

Dans cet article, nous avons déterminé deux éléments sur lesquels les amorces peuvent se construire : l'hybris et les clichés - du cliché narratif au cliché de l'expression physionomique. Mais nous savons qu'il existe d'autres éléments à partir desquels des amorces peuvent se construire car, comme nous l'avons vu, l'une des amorces étudiée dans Dexter est construite sur la succession de deux plans, c'est-à-dire sur un effet de montage. Il faudrait poursuivre cette étude afin de déterminer une typologie des amorces. Pour cela, il serait sans doute intéressant d'effectuer une étude du cliffhanger: en effet, certaines des amorces que nous avons étudiées ont lieu à ce moment précis (dans Life on Mars, le meet cute à lieu lors du cliffhanger de l'épisode 1.6 ; dans The $L$ Word, c'est au moment du cliffhanger de la saison 4 que Jodi dit à Bette "You'd better not break my heart ») - ceci parce que le cliffhanger contient intrinsèquement une amorce. Il nous reste donc à déterminer sur quels éléments autres que l'hybris et le cliché les amorces peuvent se construire.

Par ailleurs, nous avons traité ici d'amorces narratives mais il existe, à un niveau microstructural, des amorces de texte, c'est-à-dire des phrases dont on devine la suite. À ce sujet, Jennifer Hayward indique: " one particularly active soap pleasure is [...] predict plot moves (or scenes or lines) 70 ». Ainsi, dans l'épisode 2.29 de Melrose Place. Michael Mancini dit à Kimberly Shaw : "Okay, so what are you saying? » Ce à quoi Kimberly répond : «That before, we did things your way... This time, we're gonna do things my way ${ }^{71}$. " Dans cet exemple, le parallélisme guide le spectateur vers la certitude de l'épiphore. Notons que la comédienne marque évidemment une pause entre les deux parties du parallélisme. Avant que le lexème « way » soit

${ }^{68}$ Eco, De Superman au surhomme, p. 19.

69 Ibid., p. 18-19.

$7^{70}$ Jennifer Hayward, Consuming Pleasures, p. 186. "Un des plaisirs les plus forts du soap, c'est de pouvoir anticiper une action (ou une scène ou une réplique). »

${ }_{71}$ Melrose Place, épisode 2.29, minute 14. Michael : « D'accord, mais qu'est-ce que tu veux dire ? » Kimberly : «Qu'avant, on faisait les choses à ta manière ; cette fois, on va les faire à ma manière ». 
énoncé une seconde fois, le spectateur sait qu'il le sera. Le spectateur est donc dans une attitude métatextuelle, à la recherche du texte. La stratégie des amorces est une machine à multiples ressorts.

\section{Bibliographie}

Amosy Ruth et Anne Herschberg Pierrot, Stéréotypes et clichés, Paris, Armand Colin, 1997.

Eco Umberto, De Superman au surhomme, Paris, Grasset, 1993.

Eco Umberto, Lector in fabula, Paris, Grasset, 1985.

EPHRON Hallie, Writing and Selling your Mystery Novel, Writer's Digest Books, 2005.

GenetTe Gérard, Figures III, Paris, Seuil, 1992.

HAYwaRD Jennifer, Consuming Pleasures, Lexington, The University Press of Kentucky, 1997.

\section{L'auteure}

Marie Tréfousse a obtenu un Master de Langue Française, spécialité rhétorique, avec mention Très Bien à l'université Paris IV où elle a été Tutrice pendant trois ans. Son sujet de recherche portait sur le roman-feuilleton et le feuilleton télévisé, comme l'indique le titre de son mémoire de Master 1, "La Répartition de l'information ou la manipulation du récepteur », ainsi que celui de Master 2, « Le Feuilleton et son au-delà ». 